\title{
Protection against Haemophilus influenzae Type B, Diphtheria, and Tetanus in Children from Major Cities of Pakistan: A Community-Based Study
}

\author{
Muhammad Arif Nadeem Saqib ${ }^{1}$ Ibrar Rafique ${ }^{1}$ Obaid Ullah $^{2}$ Rabia Irshad $^{3}$ Najma Javed ${ }^{4}$ \\ Aftab Khan ${ }^{2}$ Rukhsana Firdous ${ }^{5}$ Ali Raza ${ }^{6}$ Muhammad Sohail Afzal ${ }^{7}{ }^{(0)}$
}

1 Pakistan Health Research Council, Islamabad, Pakistan

2 Pakistan Health Research Council Center, Khyber Medical College, Peshawar, Pakistan

3 Pakistan Health Research Council Center, Jinnah Postgraduate Medical College, Karachi, Pakistan

${ }^{4}$ Pakistan Health Research Council Center, National Institute of Health, Islamabad, Pakistan

${ }^{5}$ Pakistan Health Research Council Center, Bolan Medical College, Quetta, Pakistan

${ }^{6}$ Department of Life Sciences, School of Science, University of Management and Technology (UMT), Lahore, Pakistan

7 Department of Life Sciences, School of Science, University of

Management and Technology, Lahore, Pakistan

\author{
Address for correspondence Muhammad Sohail Afzal, PhD, \\ Department of Life Sciences, School of Science, University of \\ Management and Technology, Lahore 54000, Pakistan \\ (e-mail: sohail.ncvi@gmail.com).
}

J Pediatr Infect Dis 2020;15:163-168.

\author{
Abstract \\ Keywords \\ - pentavalent vaccine \\ - vaccine efficacy \\ - vaccine coverage \\ - Pakistan
}

Objective Pentavalent vaccine has been introduced in Pakistan against tetanus, diphtheria, Haemophilus influenzae type B ( $\mathrm{HiB})$, pertussis, and hepatitis B virus.

Methods This study was designed to determine protection against diphtheria, tetanus, and $\mathrm{HiB}$ in children of age 18 to 24 months from community which had received all three doses of pentavalent vaccine.

Results Overall, $97 \%$ were having immunity against $\mathrm{HiB}, 97 \%$ against tetanus, and $88 \%$ against diphtheria. Our study showed that children aged 18 to 24 months in Pakistan have high level of protection against $\mathrm{HiB}$ and tetanus. However, the protection for diphtheria was low in $40 \%$ of children which could be improved by adding a booster dose of diphtheria.

Conclusion By increasing the vaccine coverage among children, the burden of these diseases can be reduced in the future.

\section{Introduction}

Pakistan is a country with very high infant's mortality and morbidity. According to the World Health Organization, 393,959 children died in 2013. ${ }^{1}$ Nearly half of childhood deaths are among children younger than 5 years, compared with 8 to $10 \%$ of deaths in developed countries ${ }^{2}$ mainly because of unhygienic life style, unawareness about the infectious diseases, and poor health facilities. In Pakistan, funds allocation for health are very $\operatorname{low}^{3}$ and per capita income is negligible. ${ }^{4}$ Therefore, it is difficult for the individuals to avail good health facilities.

The major proportion of children deaths (around onethird) in Pakistan is due to vaccine preventable infectious diseases. ${ }^{1}$ To improve the health care system and ensure a better management of the infectious diseases, Pakistan government's expanded program on immunization (EPI) introduced the pentavalent vaccine in $2009,{ }^{5}$ which is provided free of cost by the government. The pentavalent vaccine has simplified the immunization activities by received

June 15, 2019

accepted

January 15, 2020

published online

February 28, 2020
Copyright @ 2020 by Georg Thieme

Verlag KG, Stuttgart . New York
DOI https://doi.org/

10.1055/s-0040-1702217. ISSN $1305-7707$. 
decreasing pain and the number of injections. The combination of vaccines has helped in getting the incorporation of other vaccines in the immunization schedule. ${ }^{6}$ The pentavalent vaccine combines five vaccines in one injection to protect against Haemophilus influenzae type B ( $\mathrm{HiB})$, diphtheria, pertussis (whooping cough), tetanus (DPT), and hepatitis $B .^{5}$ It has replaced the previous DPT and hepatitis B vaccine. Three doses of this vaccine are given to children in Pakistan during the first year, at 6, 10, and 14 weeks of age. ${ }^{2,5}$

Diphtheria is a life-threatening disease. Corynebacterium diphtheriae, a toxigenic strain, is responsible for this disease. ${ }^{7}$ The second species in this genus Corynebacterium ulcerans is also thought to be responsible for the cause of cutaneous diphtheria with the symptoms of sepsis after touching domestic cats, dogs, and foxes. ${ }^{8}$ The mode of transmission of diphtheria is through physical and respiratory contact. Diphtheria may lead to difficulties in breathing and cause death. ${ }^{7}$ In Pakistan, 45 cases of diphtheria have been reported in 2014 of which 30 cases from Punjab, 11 cases from Khyber Pakhtunkhwa (KPK), 3 cases from Sindh, and 1 case from Azad Jammu and Kashmir. ${ }^{9}$

The bacteria which are present in soil named Clostridium tetani are the cause of tetanus. The spasms and muscular stiffness are the typical symptoms of tetanus that are caused by tetanus which blocks the inhibitory neurotransmitter in the central nervous system. It is not transmitted from one individual to another. When dirt particles enter a wound or cut, then the individual becomes infected. ${ }^{10}$ Pakistan is among one of the 24 countries across the globe where maternal and neonatal tetanus is not still eliminated. Recently, it has been reported that 60 districts of Pakistan out of 139 are at high risk for tetanus infection. ${ }^{11}$

$\mathrm{HiB}$, a gram-negative bacterium, is a pathogen causing a serious disease among children all over the world. Its frequent symptoms are pneumonia and meningitis. ${ }^{12,13} \mathrm{HiB}$ is also responsible for the infections of soft tissues, epiglottis, bones, and joints. ${ }^{14} \mathrm{~A}$ recent report highlights the prevalence of $\mathrm{HiB}$ in Pakistan. Children under the age of 5 years were investigated and found that 45 children out of 1,481 were having HiB infections. ${ }^{15}$ A meta-analysis of 18 studies comparing the immunogenicity of combined vaccines versus separate vaccines showed that there was a significantly less immunological response for $\mathrm{HiB}$ and hepatitis B virus. ${ }^{16}$

Immunization is an established cost-effective strategy and public health intervention to control and eradicate the infectious diseases especially in children. Pentavalent vaccines are a combination of five vaccines against DPT, hepatitis B, and HiB. It was added in routine EPI of Pakistan in 2009. A need was felt to determine the protection level of children against these deadly diseases in our local setting as there was no information available in the literature from Pakistan at national level. The present study describes the findings of serosurvey conducted in all major cities of Pakistan to determine the protection against tetanus, $\mathrm{HiB}$, and diphtheria in children.

\section{Materials and Methods}

This was a community-based multicenter study which was conducted in four major cities (Rawalpindi, Karachi, Pesha- war, and Quetta of four provinces, i.e., Punjab, Sindh, KPK, and Balochistan, respectively) of Pakistan.

The study protocols and informed consent documents were approved by the Institutional Bioethics Review Committee of the Pakistan Health Research Council (PHRC). Informed consent for the infants was obtained from their parents or legal guardians prior to enrolling the infants in the study.

The sample size was calculated using the coverage rate reported by EPI in different regions of Pakistan. EPI has claimed 20 to $80 \%$ coverage in different regions of Pakistan. Assuming a 50\% coverage rate and by taking 5\% margin error and $95 \%$ confidence interval, the sample size of 384 was calculated for each site. However, it was done in two cities, that is, Karachi (Sindh) and Peshawar (KPK) where 384 children were enrolled, while in Rawalpindi (Punjab) and Quetta (Balochistan), 374 and 212 children were enrolled, respectively; therefore, total sample came out to be 1,354 .

Participants were selected using a multistage cluster sampling technique. Every selected city was divided into four clusters. Using a lottery method out of four, one cluster was selected. In the selected cluster, lists of households were obtained and starting from the periphery, every second house was surveyed. In case of lock or nonresponse, the next household was visited.

Healthy infants of age 18 to 24 months who had received all three doses of pentavalent vaccine were included, whereas those with recent/past history of acute or chronic illness were excluded.

After selection of the houses and taking the informed written consent from parents/guardians, a pretested questionnaire was filled which included information related to demography and vaccination. About $3 \mathrm{~mL}$ blood sample was drawn from the enrolled infants. Blood was centrifuged at $3,000 \mathrm{rpm}$ for 5 minutes and sera were separated. About $500 \mu \mathrm{l}$ of the serum was stored at $-20^{\circ} \mathrm{C}$ till analyzed.

Samples were tested for the presence of tetanus, $\mathrm{HiB}$, and diphtheria antibodies using enzyme-linked immunosorbent assay (ELISA) at PHRC Research Centers.

For determination of immunoglobulin $G(\operatorname{Ig} G)$ antibody levels for diphtheria, certified kits from DeMeditec, Germany were used having sensitivity and specificity of $94 \%$. The $\operatorname{IgG}$ antibody cutoff value considered for protection was $\geq 0.1 \mathrm{IU} / \mathrm{mL}$ for diphtheria.

For determination of IgG antibody levels for $\mathrm{HiB}$, human $\mathrm{HiB}$ IgG ELISA kit of Creative Diagnostics, United States was used having intra-assay variation $\leq 10 \%$ and interassay variation 9 to $12 \%$. The antibody concentration $<0.15 \mu \mathrm{g} / \mathrm{mL}, 0.15$ showed insufficient protection against $\mathrm{HiB}$. However, the antibody concentration between 0.15 to 1.0 and $>1.0 \mu \mathrm{g} / \mathrm{mL}$ indicated immunization with polyribosyl ribitol phosphate (PRP) and sufficient natural immunity (SNI) or an acquired protection after the third vaccination, respectively.

For determination of IgG antibody levels for tetanus, certified kits from Vircell, Spain were used with 96\% sensitivity and specificity. Samples with indexes above 11 were considered as having IgG-specific antibodies against tetanus. The index was calculated as (sample optical density [OD]/ 
cutoff serum mean OD) $\times 10$. Positive, negative, and cutoff controls were provided with the kit.

Standards and test serum samples were tested in duplicate. The absorbance was measured with an ELISA reader at $450 \mathrm{~nm}$. The mean OD values were calculated from two readings of each sample. The validation criteria mentioned in kit literature was strictly adhered to. From the OD values of standards, a reference curve was plotted, and finally, the concentration of $\operatorname{IgG}$ antibody was determined for each sample. The serological testing was performed at the PHRC Research Centers of Peshawar and Karachi following Good Laboratory Practice guidelines.

\section{Results}

A total of 1,294 children were enrolled including 350 from Peshawar and Karachi each, while 348 from Rawalpindi and 212 from Quetta. Among all, 771 (57\%) were males and 583 (43\%) were females.

For $\mathrm{HiB}$, overall $97 \%$ were immune against the disease and among them, $56 \%$ were had SNI, while 41 showed antibodies against vaccine, that is, immunized with polyribosyl ribitol phosphate (IPRP). The remaining 3\% did not have either SNI or antibodies against IPRP making them vulnerable to disease.

Within the cities, the children belonging to Quetta city (Balochistan) had highest protection, that is, $96 \%$ (SNI) against HiB followed by $61 \%$ in Karachi (Sindh), $41 \%$ in Peshawar (KPK) and Rawalpindi (Punjab) each. Majority (57\%) were IPRP in Peshawar followed by $51 \%$ in Rawalpindi, $38 \%$ in Karachi, and $41.1 \%$ in Quetta. No age-specific difference regarding $\mathrm{HiB}$ has been observed in this study ( $\mathbf{-}$ Tables $\mathbf{1 - 3}$ ).

Overall $96 \%$ were positive for tetanus, and the rests were negative and/or equivocal. When compared between cities, 99\% were positive in Peshawar, 98\% in Quetta, and 97\% in Rawalpindi followed by $92 \%$ in Karachi. Almost 6\% were found to be not protected in Karachi which is higher as compared with other cities. There was no significant variation with the age (-Tables $1-3$ ).

Regarding diphtheria, overall $63 \%$ were fully protected, $25 \%$ were somewhat protected, while $12 \%$ were not pro-
Table 2 Haemophilus influenzae type B within 18 to 24 months age group

\begin{tabular}{|l|l|l|l|l|l|l|}
\hline $\begin{array}{l}\text { Age } \\
(\mathbf{m o})\end{array}$ & Number & SNI & IPRP & ISNI & TP & $\%$ \\
\hline 18 & 268 & 162 & 99 & 4 & 261 & 97 \\
\hline 19 & 94 & 62 & 31 & 1 & 93 & 99 \\
\hline 20 & 138 & 86 & 47 & 5 & 133 & 96 \\
\hline 21 & 71 & 52 & 18 & 1 & 70 & 99 \\
\hline 22 & 81 & 53 & 27 & 1 & 80 & 99 \\
\hline
\end{tabular}

Abbreviations: IPRP, immunized with polyribosyl ribitol phosphate; ISNI, insufficient natural immunity; SNI, sufficient natural immunity; TP, total (overall) protection.

tected. In the case of Quetta, the majority (92\%) were fully protected followed by almost 62,57 , and $56 \%$ in Karachi, Rawalpindi, and Peshawar, respectively. About 36\% were somewhat protected against diphtheria in Karachi followed by $19.3 \%$ in Rawalpindi. Almost 23\% in Peshawar and $21 \%$ in Rawalpindi were not protected against diphtheria. Also, the protection level showed a downward trend with an increase in age, but this was not consistent ( - Table 4).

\section{Discussion}

The dire need of the day is the prevention of such diseases which are preventable by vaccines. It has been observed that every year approximately three millions of children die globally from vaccine preventable diseases. ${ }^{17}$ Over the past two decades, DPT vaccination coverage has been improved in Pakistan. The DPT trivalent vaccination coverage was $35 \%$ in $1990-1991$, then it has been increased to $59 \%$ in 2006-2007. ${ }^{18}$ The recent Pakistan demographic health survey reported that coverage of three doses of trivalent vaccine was $65 \%$ in 2012-2013. The information was taken from both vaccination cards and mother sources. The proportion of coverage, however, varies within cities. The coverage of three doses of trivalent vaccine was $76 \%$ in Punjab followed by 69.6\% in KPK, 38.6\% in Sindh, and 27\% in Balochistan. More male children were vaccinated than female children. ${ }^{18}$ The

Table $1 \mathrm{HiB}$, tetanus, and diphtheria in different cities of Pakistan

\begin{tabular}{|l|l|l|l|l|l|l|l|l|l|}
\hline \multirow{3}{*}{ Province/city } & \multicolumn{4}{l|}{ HiB } & \multicolumn{3}{l|}{ Tetanus } & \multicolumn{2}{l|}{ Diphtheria } \\
\cline { 2 - 10 } & SNI & IPRP & $\begin{array}{l}\text { ISNI } \\
\text { N (\%) }\end{array}$ & $\begin{array}{l}\text { Positive } \\
N(\%)\end{array}$ & $\begin{array}{l}\text { Negative } \\
N(\%)\end{array}$ & $\begin{array}{l}\text { Equivocal } \\
N(\%)\end{array}$ & $\begin{array}{l}\text { FP } \\
N(\%)\end{array}$ & $\begin{array}{l}\text { SP } \\
N(\%)\end{array}$ & $\begin{array}{l}\text { NP } \\
N(\%)\end{array}$ \\
\hline Punjab/Rawalpindi & 141 & 178 & 29 & 340 & 2 & 6 & 208 & 67 & 73 \\
& $(40.5 \%)$ & $(51.1 \%)$ & $(8.3 \%)$ & $(97.7 \%)$ & $(0.6 \%)$ & $(1.7 \%)$ & $(59.8 \%)$ & $(19.3 \%)$ & $(21 \%)$ \\
\hline Sindh/Karachi & 236 & 146 & 2 & 352 & 23 & 9 & 237 & 139 & 8 \\
& $(61.5 \%)$ & $(38 \%)$ & $(0.5 \%)$ & $(91.7 \%)$ & $(6 \%)$ & $(2.3 \%)$ & $(61.7 \%)$ & $(36.2 \%)$ & $(2.1 \%)$ \\
\hline KPK/Peshawar & 143 & 200 & 7 & 348 & 2 & 0 & 209 & 58 & 83 \\
& $(41 \%)$ & $(57 \%)$ & $(2 \%)$ & $(99 \%)$ & $(1 \%)$ & 0 & $(60 \%)$ & $(17 \%)$ & $(23 \%)$ \\
\hline Balochistan/Quetta & 204 & 532 & 38 & 207 & 5 & 0 & 194 & 17 & 1 \\
& $(96 \%)$ & $(41.1 \%)$ & $(2.9 \%)$ & $(98 \%)$ & $(2 \%)$ & 0 & $(92 \%)$ & $(8 \%)$ & $(0 \%)$ \\
\hline
\end{tabular}

Abbreviations: FP, fully protected; HiB, Haemophilus influenzae type B; IPRP, immunized with polyribosyl ribitol phosphate; ISNI, insufficient natural immunity; KPK, Khyber Pakhtunkhwa; NP, not protected; SNI, sufficient natural immunity; SP, somewhat protected. 
Table 3 Tetanus in 18 to 24 months age group

\begin{tabular}{|l|l|l|l|l|l|l|}
\hline Age $(\mathbf{m o})$ & Number & Positive & Negative & Equivocal & TP & $\%$ \\
\hline 18 & 268 & 260 & 4 & 4 & 264 & 99 \\
\hline 19 & 94 & 91 & 3 & 0 & 91 & 97 \\
\hline 20 & 138 & 135 & 3 & 0 & 135 & 98 \\
\hline 21 & 71 & 70 & 1 & 0 & 70 & 99 \\
\hline 22 & 81 & 78 & 1 & 2 & 80 & 99 \\
\hline 23 & 106 & 104 & 2 & 0 & 104 & 98 \\
\hline 24 & 536 & 510 & 17 & 9 & 519 & 97 \\
\hline
\end{tabular}

Abbreviation: TP, total (overall) protection.

Table 4 Diphtheria in 18 to 24 months age group

\begin{tabular}{|l|l|l|l|l|l|l|}
\hline Age (mo) & Kids & FP & SP & NP & TP & $\%$ \\
\hline 18 & 268 & 192 & 52 & 24 & 244 & 91 \\
\hline 19 & 94 & 72 & 18 & 4 & 90 & 96 \\
\hline 20 & 138 & 96 & 21 & 21 & 117 & 85 \\
\hline 21 & 71 & 54 & 8 & 9 & 62 & 87 \\
\hline 22 & 81 & 58 & 15 & 9 & 73 & 90 \\
\hline 23 & 106 & 69 & 21 & 16 & 90 & 85 \\
\hline 24 & 536 & 307 & 151 & 78 & 458 & 85 \\
\hline
\end{tabular}

Abbreviation: FP, fully protected; NP, not protected; SP, somewhat protected; TP, total (overall) protection.

pentavalent vaccine was introduced in Pakistan in 2009, and currently, there is no data regarding the efficacy of the pentavalent vaccine. So, this study was performed nationwide and the results of pentavalent vaccine immunization against tetanus, diphtheria, and $\mathrm{HiB}$ are 97,88 , and 97\%, respectively.

Various combination vaccines are in practice globally to prevent DPT. ${ }^{19}$ Vaccination against tetanus has depicted a major decline in the case of this fatal disease. ${ }^{20}$ By conducting programs of effective immunization and good postexposure prophylaxis, tetanus is rare in developed countries. ${ }^{21,22}$ A recent report from Poland regarding the immunization status against tetanus in vaccinated children (number $=324$; aged $18-180$ months) showed that $29 \%$ of children were protected, $5.86 \%$ were unprotected, and $65.12 \%$ were considered as an intermediate. ${ }^{23}$ A study from United Arab Emirates regarding the tetanus vaccine efficacy in a group of 227 children showed that $89.9 \%$ of children were protected, $4 \%$ showed lack of protection, and $2.2 \%$ were equivocal against tetanus. ${ }^{24}$ From Gaza, Al Aswad reported that the protection of tetanus from the vaccine was $95 \%$ out of 180 children. ${ }^{25}$ Our results regarding the immunization status against tetanus by using the pentavalent vaccine showed that regarding tetanus, 99\% were protected against this disease in Punjab and KPK, 98\% in Balochistan, and 94\% in Sindh. Similar findings were also reported from Iran (96\%) and Gaza (98\%). ${ }^{25-27}$ The exact incidence of tetanus across the globe is unknown and it is estimated that only $10 \%$ of cases are reported. ${ }^{28}$ In 2013, seven cases of tetanus were reported in the United Kingdom, but it is more common in other parts of the world. ${ }^{29}$

A significant decline has been observed in the case of diphtheria due to vaccination. Before the introduction of routine vaccination, $\sim 1,800$ deaths were recorded related to diphtheria each year. ${ }^{30}$ Diphtheria antibodies $(\operatorname{IgG})$ can be induced by natural toxin during clinical or subclinical infection and by immunization. ${ }^{31,32}$ In this study, 63\% were fully protected against diphtheria. A similar finding was reported by studies conducted in Islamabad and Israel where fully protected children against diphtheria were $74 \%{ }^{33,34}$ The results showed that the proportion of "nonprotected" children was highest in Punjab and the same were the findings of a study from India where seroprotection was also around $50 \%{ }^{34}$ A similar finding was reported recently from Poland where the seroprotection against diphtheria was only $29.32 \%{ }^{23}$ Our findings were however contrary to those reported from Iran, United Arab Emirates, and Palestine where seroprotection rate against diphtheria was 99, 86, and $87 \%$, respectively. $24,26,27$

Since the early 1990s, there has been a gradual introduction of the $\mathrm{HiB}$ conjugate vaccine into more and more routine programs. In 2004, 89 countries used the vaccine, but in 2011 , the number of countries has increased up to $172 .{ }^{35}$ Before the introduction of vaccines, there was a large number of cases of $\mathrm{HiB}$ in different regions and 20,000 cases were estimated annually from the United States. ${ }^{36}$ The efficacy of the $\mathrm{HiB}$ vaccine should also be under observation along with the progress of vaccination. Different reports are available on 
the efficacy of $\mathrm{HiB}$ vaccine from different countries. The results of this study showed that overall $97 \%$ of children were protected against $\mathrm{HiB}$ which is similar to the findings of the studies reported from Salvador, India, and England. ${ }^{36-38}$

\section{Conclusion}

There are several diseases that have been eradicated globally by the introduction of vaccines. Although the vaccination preparation and scheduling of vaccines advanced, several vaccine preventable diseases still exist. In Pakistan, the infant's death rate is still higher than the other developing countries. From the past two decades, the vaccination coverage has been improved in Pakistan, which results in decreased infant death rate. The current study showed high level of protection against tetanus and $\mathrm{HiB}$ in children aged 18 to 24 months. However, the protection against diphtheria was low in $40 \%$ children; therefore, a booster dose for diphtheria at 18 months needs to be added in routine EPI. This will eventually improve the infant's immunization and will reduce the mortality and morbidity against vaccine preventable diseases.

Note

The study protocols and informed consent documents were approved by the Institutional Bioethics Review Committee (IBRC) of Pakistan Health Research Council (PHRC). For infants, informed consent to participate in the study was obtained from their parents or legal guardians prior to enrolling the infants in the study. Informed written consent for publication was obtained from each participant which stated that the details/images/videos will be freely available on the internet and may be seen by the general public.

\section{Availability of Data and Materials}

The datasets used and/or analyzed during the current study are available from the corresponding author on reasonable request.

\section{Authors' Contributions}

M.A.N.S. and I.R. designed the study. O.U., R.I., N.J., A.K., and R.F. collected the data. M.A.N.S., I.R., A.R., and M.S.A. did the data analysis and wrote the manuscript.

\section{Funding}

The study was funded by Pakistan Health Research Council.

\section{Conflict of Interest}

None declared.

\section{Acknowledgment}

We sincerely acknowledge efforts of the PHRC research centers in collection of the data.

\section{References}

1 UNICEF/WHO/The World Bank/UN Pop Div. Levels and Trends in Child Mortality. Report 2019. Available at: https://www.unicef.
org/media/60561/file/UN-IGME-child-mortality-report-2019.pdf. Accessed February 18, 2020

2 Childhood Immunization in Pakistan. RESEARCH AND DEVELOPMENT SOLUTIONS Policy Briefs Series No.3, February 2012. Available at: http://resdev.org/files/policy_brief/3/3.pdf. Accessed March 21, 2017

3 Ahmed J, Shaikh BT. An all time low budget for healthcare in Pakistan. J Coll Physicians Surg Pak 2008;18(06):388-391

4 Pakistan Economic Survey 2015-16. Economic Adviser's Wing, Finance Division, Government of Pakistan, Islamabad. Available at: http://www.irispunjab.gov.pk/Economic\%20Surveys-New/ Economic\%20Survey\%202015-16.pdf. Accessed May 23, 2017

5 Immunization in Pakistan. Available at: http://www.pildat.org/ publications/publication/publichealth/Immunizationinpakistan. pdf. Accessed September 8, 2015

6 Nakayama T, Aizawa C, Kuno-Sakai H. A clinical analysis of gelatin allergy and determination of its causal relationship to the previous administration of gelatin-containing acellular pertussis vaccine combined with diphtheria and tetanus toxoids. J Allergy Clin Immunol 1999;103(2 Pt 1):321-325

7 Diphtheria Reported cases. Available at: http://apps.who.int/ immunization_monitoring/globalsummary/timeseries/tsincidencediphtheria.html. Accessed May 23, 2015

8 Moore LSP, Leslie A, Meltzer M, Sandison A, Efstratiou A, Sriskandan S. Corynebacterium ulcerans cutaneous diphtheria. Lancet Infect Dis 2015;15(09):1100-1107

9 SEASONAL AWARENESS AND ALERT LETTER. For Epidemic prone Infectious Diseases in Pakistan. Available at: http://202.83.164.138/userfiles/file/nhsrc\%20pdf\%20files/SAAL\% 20News\%20Letter.pdf. Accessed May 23, 2017

10 Current recommendations for treatment of tetanus during humanitarian emergencies. WHO Technical Note January 2010. Available at: http://www.who.int/diseasecontrol_emergencies/ who_hse_gar_dce_2010_en.pdf. Accessed May 19, 2017

11 Khan EA, Khan R, Iqbal MT, et al. Risk characterization of maternal and neonatal tetanus in view of tetanus vaccination campaigns in Pakistan. J Ayub Med Coll Abbottabad 2015;27 (01):220-222

12 Review panel on Haemophilus influenzae type b (Hib) disease burden in Bangladesh, Indonesia and other Asian countries, Bangkok, 28-29 January 2004. Wkly Epidemiol Rec 2004;79 (18):173-175

13 Bryce J, Boschi-Pinto C, Shibuya K, et al and WHO Child Health Epidemiology Reference Group. WHO estimates of causes of death in children. Lancet 2005;365:1147-1152

14 Watt JP, Wolfson LJ, O'Brien KL, et al; Hib and Pneumococcal Global Burden of Disease Study Team. Burden of disease caused by Haemophilus influenzae type $b$ in children younger than 5 years: global estimates. Lancet 2009;374(9693):903-911

15 Zaidi AK, Khan H, Sherali AR, Lasi R; Sindh Meningitis Study Group. Burden of Haemophilus influenzae type $\mathrm{b}$ disease in Pakistani children. East Mediterr Health J 2010;16(06):590-594

16 Bar-On ES, Goldberg E, Fraser A, Vidal L, Hellmann S, Leibovici L. Combined DTP-HBV-HIB vaccine versus separately administered DTP-HBV and HIB vaccines for primary prevention of diphtheria, tetanus, pertussis, hepatitis B and Haemophilus influenzae B (HIB). Cochrane Database Syst Rev 2009;(03):CD005530

17 Shafi K, Nawab F. Vaccination practices of children under two years of age, admitted in tertiary care hospital of Karachi, Pakistan. ASH \& KMDC 2016;21(01):23-28

18 Pakistan Demographic and Health Survey (PDHS) 2012-13. Available at: http://www.nips.org.pk/abstract_files/PDHS\%20Final\%20Report\% 20as\%20of\%20Jan\%2022-2014.pdf. Accessed May 20, 2017

19 Information Sheet by WHO. Observed rate of vaccine reaction diphtheria, pertussis, tetanus vaccines. Available at: http://www. who.int/vaccine_safety/initiative/tools/DTP_vaccine_rates_information_sheet.pdf. Accessed March 30, 2017 
20 Hammarlund E, Thomas A, Poore EA, et al. Durability of vaccine-induced immunity against tetanus and diphtheria toxins: a cross-sectional analysis. Clin Infect Dis 2016;62 (09):1111-1118

21 Verde F, Riboldi G, Zappa C, et al. An old woman with pressure ulcer, rigidity, and opisthotonus: never forget tetanus!. Lancet 2014;384(9961):2266

22 Alves M, Canoui E, Deforges L, et al. An unexpected trismus. Lancet 2012;380(9840):536

23 Gowin E, Wysocki J, Kałużna E, et al. Does vaccination ensure protection? Assessing diphtheria and tetanus antibody levels in a population of healthy children: a cross-sectional study. Medicine (Baltimore) 2016;95(49):e5571

24 Al-Mekaini LA, Kamal SM, Al-Jabri O, et al. Seroprevalence of vaccine-preventable diseases among young children in the United Arab Emirates. Int J Infect Dis 2016;50:67-71

25 Aswad A, Hassan I, Shubair M. Efficacy of diphtheria and tetanus vaccination in Gaza strip. 2006. Available at: http://hdl.handle. net/20.500.12358/21700. Accessed February 18, 2020

26 Eslamifar A, Ramezani A, Banifazl M, et al. Immunity to diphtheria and tetanus among blood donors in Arak, central province of Iran. Iran J Microbiol 2014;6(03):190-193

27 Al Aswad IH, Shubair ME. Efficacy of diphtheria and tetanus vaccination in Gaza, Palestine. East Mediterr Health J 2009;15 (02):285-294

28 Sutter RW, Cochi SL, Brink EW, Sirotkin BI. Assessment of vital statistics and surveillance data for monitoring tetanus mortality, United States, 1979-1984. Am J Epidemiol 1990;131(01): 132-142

29 Public Health England. Tetanus in England and Wales: 2013. Available at: https://www.gov.uk/government/publications/tetanusin-england-and-wales-2013/tetanus-in-england-and-wales2013. Accessed October 2015
30 Roush SW, Murphy TV; Vaccine-Preventable Disease Table Working Group. Historical comparisons of morbidity and mortality for vaccine-preventable diseases in the United States. JAMA 2007; 298(18):2155-2163

31 Damasco PV, Pimenta FP, Filardy AA, et al. Prevalence of IgG diphtheria antitoxin in blood donors in Rio de Janeiro. Epidemiol Infect 2005;133(05):911-914

32 McQuillan GM, Kruszon-Moran D, Deforest A, Chu SY, Wharton M. Serologic immunity to diphtheria and tetanus in the United States. Ann Intern Med 2002;136(09):660-666

33 Faryal R, Noreen Z, Tahir F, Rehman Z. Report: seroprevalence of corynebacterium diphtheriae among vaccinated population of Rawalpindi/Islamabad, Pakistan. Pak J Pharm Sci 2013;26(03): 649-651

34 Sharma HJ, Yadav S, Lalwani SK, et al. Immunogenicity and safety of an indigenously manufactured reconstituted pentavalent (DTwP-HBV + Hib) vaccine in comparison with a foreign competitor following primary and booster immunization in Indian children. Hum Vaccin 2011;7(04):451-457

35 WHO. Vaccine-preventable disease monitoring system. Global and regional immunization profile. Available at: http://wwwwhoint/ immunization_monitoring/en/globalsummary/GS_GLOProfilepdf. Accessed December 2011

36 Peltola H. Haemophilus influenzae type b disease and vaccination in Latin America and the Caribbean. Pediatr Infect Dis J 1997;16 (08):780-787

37 Suárez E, Asturias EJ, Hilbert AK, Herzog C, Aeberhard U, Spyr C. A fully liquid DTPw-HepB-Hib combination vaccine for booster vaccination of toddlers in El Salvador. Rev Panam Salud Publica 2010;27(02):117-124

38 Ladhani S, Ramsay M, Flood J, et al. Haemophilus influenzae serotype B (Hib) seroprevalence in England and Wales in 2009. Euro Surveill 2012;17(46):20313 